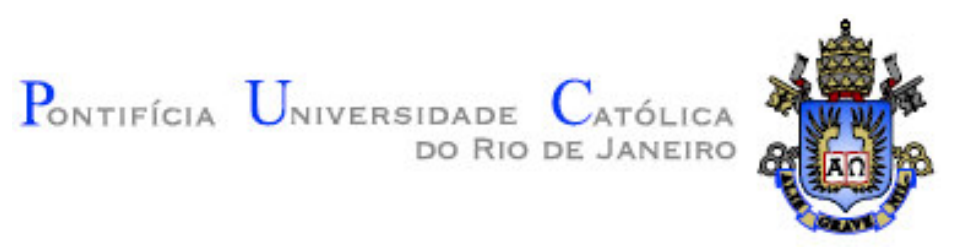

João Felipe Barbosa Alves

\title{
Uma Análise Comparativa dos Principais Métodos Baseados em Partículas para Simulação de Escoamentos
}

Dissertação de Mestrado

Dissertação apresentada como requisito parcial para obtenção do título de Mestre pelo Programa de Pós-Graduação em Engenharia Mecânica da PUCRio.

Orientador: Prof. Marcelo de Andrade Dreux 


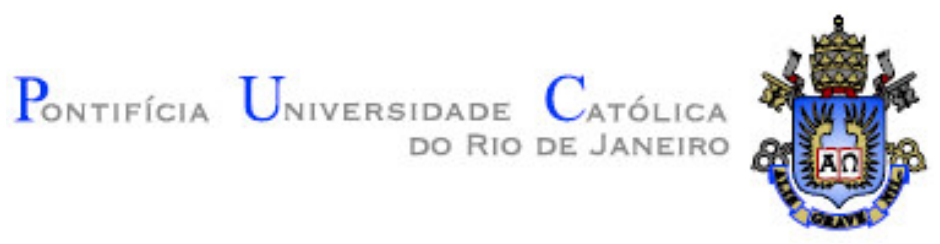

João Felipe Barbosa Alves

\title{
Uma Análise Comparativa dos Principais Métodos Baseados em Partículas para Simulação de Escoamentos
}

\begin{abstract}
Dissertação apresentada como requisito parcial para obtenção do título de Mestre pelo Programa de PósGraduação em Engenharia Mecânica da PUC-Rio. Aprovada pela Comissão Examinadora abaixo assinada.
\end{abstract}

Prof. Marcelo de Andrade Dreux Orientador Departamento de Engenharia Mecânica - PUC-Rio

Profa. Angela Ourivio Nieckele Departamento de Engenharia Mecânica - PUC-Rio

Prof. Bruno Feijó Departamento de Informática - PUC-Rio

Prof. José Eugênio Leal Coordenador(a) Setorial do Centro Técnico Científico - PUC-Rio 
Todos os direitos reservados. É proibida a reprodução total ou parcial do trabalho sem autorização da universidade, do autor e do orientador.

\title{
João Felipe Barbosa Alves
}

Graduou-se em Engenharia Química pela Escola de Química da Universidade Federal do Rio de Janeiro. Durante o mestrado, foi bolsista $\mathrm{CNPq}$ e pesquisador no Laboratório de Computação Gráfica, Tecgraf, em projeto financiado pela Petrobrás.

Ficha Catalográfica

\begin{abstract}
Alves, João Felipe Barbosa
Uma Análise Comparativa dos Principais Métodos Baseados em Partículas para Simulação de Escoamentos / João Felipe Barbosa Alves; orientador: Marcelo de Andrade Dreux. - 2008.
\end{abstract}

96f. : il. ; $30 \mathrm{~cm}$

Dissertação (Mestrado em Engenharia Mecânica) Pontifícia Universidade Católica do Rio de Janeiro, Rio de Janeiro, 2008.

Inclui bibliografia.

1. Engenharia Mecânica - Teses. 2. Simulação de Escoamentos. 3. MPS. 4. SPH. 5. Método de Partículas. I. Dreux, Marcelo de Andrade. II. Pontifícia Universidade Católica do Rio de Janeiro. Departamento de Engenharia Mecânica. III. Título.

CDD: 621 


\section{Agradecimentos}

Ao meu orientador professor Marcelo de Andrade Dreux e ao Dr. Ernesto Fleck pelo apoio e orientação durante o desenvolvimento deste trabalho.

Aos meus pais, que sempre me incentivaram e me deram a oportunidade de estudar.

À professora Angela O. Nieckele pelo auxílio na utilização do software Fluent ${ }^{\circledR}$.

Agradeço a Sra. Denise Fleck pelas palavras de incentivo e conselhos no início do trabalho.

Ao M. Eng. Márcio Michiaru Tsukamoto do laboratório Tanque de Provas Numérico (TPN) do departamento de Engenharia Naval e Oceânica da Escola politécnica da Universidade de São Paulo, pela valiosa ajuda durante a implementação do método MPS.

A minha família e amigos, que sempre torceram pelo meu sucesso.

Um agradecimento especial à Julia, minha noiva, por estar sempre ao meu lado nos momentos em que eu mais precisei.

Ao CNPq e à PUC-Rio, pelos auxílios concedidos. 


\section{Resumo}

Alves, João Felipe Barbosa; Dreux, Marcelo de Andrade. Uma Análise Comparativa dos Principais Métodos Baseados em Partículas para Simulação de Escoamentos. Rio de Janeiro, 2008. 96p. Dissertação de Mestrado - Departamento de Engenharia Mecânica, Pontifícia Universidade Católica do Rio de Janeiro.

Neste trabalho, foi realizado um estudo comparativo de eficiência e acurácia dos métodos de partículas Moving Particle Semi-implicit Method (MPS) e Smoothed Particle Hydrodynamics (SPH). A acurácia dos métodos de partículas foi determinada tomando-se como referência os métodos dos Volumes Finitos e Volume of Fluid (VOF). A comparação de acurácia entre os métodos MPS e SPH foi realizada através da simulação dos problemas de quebra de barragem e de descarga de água. Além disso, o problema de escoamento laminar em uma cavidade quadrada e o problema do tubo de choque foram simulados com sucesso pelo método SPH. A análise de eficiência foi realizada pela determinação do tempo total de processamento em função do número de partículas. Adicionalmente, uma análise da influencia do número de partículas na solução foi realizada. Os resultados obtidos mostram que ambos os métodos podem ser considerados como boas ferramentas para a simulação de fluidos.

\section{Palavras-chave}

Simulação do escoamento de fluidos; MPS; SPH; Método de partícula; DFC. 


\section{Abstract}

Alves, João Felipe Barbosa. Dreux, Marcelo de Andrade. A Comparative Analysis of the Main Particle-Based Methods Used for Flow Simulation. Rio de Janeiro, 2008. 96p. MSc. Dissertation - Departamento de Engenharia Mecânica, Pontifícia Universidade Católica do Rio de Janeiro.

This work comprises a comparative study of the particle methods Moving Particle Semi-implicit (MPS) and Smoothed Particle Hydrodynamics (SPH) in terms of their efficiency and accuracy. The methods of Finite Volume and Volume of Fluid (VOF) were used as reference for determining the accuracy of the particle methods. The methods MPS and SPH were compared with each other by means of simulations of the problems of dam collapse and water discharge. On top of that, the problems of shear driven cavity and shock tube were successfully simulated using SPH. In order to analyze the methods' efficiency, the total processing time as a function of the number of particles was calculated. Finally, an analysis of the influence of the number of particles in solution was performed. The results obtained in this work show that both the MPS and SPH methods can be considered as good tools for fluid simulation.

\section{Keywords}

Fluid simulation; MPS; SPH; Particle method; CFD. 


\section{Sumário}

1 Introdução 13

1.1. Revisão da Literatura $\quad 15$

1.2. Estrutura da Dissertação 21

2 Equações Governantes e Formalismo Básico dos Métodos de Partículas 22

2.1. Equações Governantes 22

2.1.1. Equação da Continuidade 22

2.1.2. Equação da Quantidade de Movimento 23

2.1.3. Equação de Energia

2.2. Formalismo Básico dos Métodos de Partículas 26

3 O Método de Partículas MPS 29

3.1. Função Peso 29

3.2. Densidade do Número de Partículas 30

3.3. Modelo do Operador Gradiente 31

3.4. Modelo do Operador Laplaciano 33

3.5. Incompressibilidade 34

3.6. Superfície Livre 36

3.7. Implementação do Método MPS 36

3.7.1. Tipos de Partículas 36

3.7.2. Determinação do Raio de Vizinhança 37

3.7.3. Determinação do Passo de Tempo 37

3.7.4. Determinação do Parâmetro $\lambda \quad 38$

3.7.5. Tratamento de Colisões 38

3.7.6. O algoritmo MPS 40

3.7.7. Visualização dos Resultados 41

3.8. Apresentação dos Resultados 41

3.8.1. Escoamento de Fluido Incompressível e Não Viscoso 42

4 O Método de Partículas SPH 46 
4.1. Aproximação por Partículas 46

4.2. Funções de Suavização 48

4.3. Aplicação do Método SPH na Modelagem de Fluidos 52

4.3.1. Determinação da Densidade 53

4.3.2. Determinação do Gradiente de Pressão 54

4.3.3. Viscosidade $\quad 57$

4.3.4. Viscosidade Artificial $\quad 58$

4.3.5. Forças Externas e de Corpo 59

4.3.6. Determinação da Energia Interna $\quad 59$

4.4. Implementação do Método SPH 60

4.4.1. Atributos Associados às Partículas $\quad 60$

4.4.2. Determinação do Domínio de Suporte $k h \quad 60$

4.4.3. Determinação do Passo de Tempo 61

4.4.4. Tratamento de Fronteiras $\quad 61$

4.4.5. Evolução das Partículas

4.4.6. O Algoritmo SPH 63

4.5. Apresentação dos Resultados 64

4.5.1. Escoamento de Fluido Compressível e Não Viscoso 64

4.5.2. Escoamento de Fluido Incompressível e Viscoso 67

4.5.3. Escoamento de Fluido Incompressível e Não Viscoso 71

5 Análise dos Resultados $\quad 75$

5.1. Análise de Acurácia entre os Métodos MPS e SPH 75

5.2. Análise de Eficiência entre os Métodos MPS e SPH 82

5.3. Influência do Número de Partículas na Solução 84

6 Conclusão $\quad 88$

7 Bibliografia $\quad 90$ 


\section{Lista de figuras}

Figura 1 - Trabalho de Monaghan (1994). Simulação do problema de quebra de barragem. Imagens extraídas de Monaghan (1994).

Figura 2 - Trabalho de Ashtiani e Farhadi (2006). Simulação do problema de quebra de barragem. Imagem extraída de Ashtiani e Farhadi (2006).

Figura 3 - Trabalho de Nakamura (2007). Simulação do problema de descarga de água. Imagens extraídas de Nakamura (2007).

Figura 4 - Função peso utilizada pelo método MPS (Koshizuka e Oka, 1996).

Figura 5 - Representação esquemática da distribuição de partículas (adaptado de Tsukamoto, 2006).

Figura 6 - Conceito do modelo do operador gradiente (adaptado de Koshizuka et al., 1998a).

Figura 7 - Conceito do modelo do operador laplaciano (adaptado de Koshisuka et al., 1998a).

Figura 8 - Representação esquemática do algoritmo básico do método MPS (adaptado de Tovar et al, 2005).

Figura 9 - Modelo do problema de quebra de barragem (adaptado de Koshizuka e Oka, 1996).

Figura 10 - Resultado da simulação do problema de quebra de barragem utilizando o método MPS.

Figura 11 - Modelo do problema de descarga de água.

Figura 12 - Resultado da simulação do problema de descarga de água utilizando o método MPS.

Figura 13 - Função de suavização spline cúbica e sua primeira derivada (função traçada com raio de suavização igual a um).

Figura 14 - Função de suavização spline de quinta ordem e sua primeira derivada (função traçada com raio de suavização igual a um).

Figura 15 - Representação esquemática do algoritmo básico do método SPH.

Figura 16 - Perfil de densidade no tubo de choque no instante $t=0,15$

s. A onda de choque é observada próxima ao ponto $x=0,2 \mathrm{~m}$. 
Figura 17 - Perfil de pressão no tubo de choque no instante $t=0,15$ s. A onda de choque é observada próxima ao ponto $x=0,2 \mathrm{~m}$.

Figura 18 - Modelo do problema de escoamento laminar de um fluido contido no interior de uma cavidade quadrada.

Figura 19 -Distribuição inicial das partículas no problema de escoamento laminar em uma cavidade quadrada. As partículas virtuais não estão apresentadas.

Figura 20 - Malha gerada pelo software Gambit ${ }^{\circledR}$ para o problema de escoamento laminar em uma cavidade quadrada.

Figura 21 - Variação da velocidade adimensional vertical ao longo de uma linha central horizontal.

Figura 22 - Variação da velocidade adimensional horizontal ao longo de uma linha central vertical.

Figura 23 - Vetores de velocidade para o problema de escoamento laminar em uma cavidade quadrada na condição de regime permanente obtida pelo método SPH. A escala de cores e o tamanho dos vetores indicam a magnitude da velocidade em metros por segundo. Figura 24 - Vetores de velocidade para o problema de escoamento laminar em uma cavidade quadrada na condição de regime permanente obtida pelo método dos Volumes Finitos. A escala de cores e o tamanho dos vetores indicam a magnitude da velocidade em metros por segundo. Figura 25 - Resultado da simulação do problema de quebra de barragem utilizando o método SPH.

Figura 26 - Simulação do problema de descarga de água pelo método SPH.

Figura 27 - Malha usada pelo Fluent na simulação do problema de quebra de barragem.

Figura 28 - Comparação entre os métodos VOF, MPS e SPH no problema de quebra de barragem.

Figura 29 - Movimentação da frente do escoamento no problema de quebra de barragem. Neste gráfico estão apresentados os pontos e o ajuste dos mesmos em um polinômio de terceiro grau. Os símbolos g e t representam a aceleração da gravidade e o tempo, respectivamente. Figura 30 - Comparação entre os métodos VOF, MPS e SPH no problema de quebra de barragem após o fluido encontrar a parede vertical da direita. 
Figura 31 - Comparação dos resultados obtidos com os métodos MPS e SPH na simulação do problema de quebra de barragem com o experimento realizado por Koshizuka e Oka (1996).

Figura 32 - Malha usada pelo Fluent para o problema de descarga de água.

Figura 33 - Comparação entre os métodos VOF, MPS e SPH no problema de descarga de água.

Figura 34 - Variação do nível no interior da comporta no problema de descarga de água. Neste gráfico estão apresentados os pontos e o ajuste dos mesmos em um polinômio de quarto grau. Os símbolos g e t representam a aceleração da gravidade e o tempo, respectivamente. Figura 35 - Movimentação da frente do escoamento no problema de descarga de água. Neste gráfico estão apresentados os pontos e o ajuste dos mesmos em um polinômio de quarto grau. Os símbolos g e t representam a aceleração da gravidade e o tempo, respectivamente. Figura 36 - Análise do custo computacional em função do número de partículas de fluido dos métodos MPS e SPH. O tempo total da simulação do problema foi de um segundo.

Figura 37-Gráfico em barras da variação do tempo total de processamento em função do número de partículas de fluido na simulação do problema de quebra de barragem pelos métodos MPS e SPH. As simulações foram realizadas com 648, 882, 1152 e 1682 partículas de fluido.

Figura 38 - Influência do número de partículas na solução do problema de quebra de barragem utilizando-se o método MPS. As curvas representam o ajuste dos pontos em um polinômio de terceiro grau. Figura 39 - Influência do número de partículas na simulação do problema de quebra de barragem utilizando-se o método MPS. O escoamento já atingiu a parede vertical da direita.

Figura 40 - Influência do número de partículas na solução do problema de quebra de barragem utilizando-se o método SPH. As curvas representam o ajuste dos pontos em um polinômio de terceiro grau.

Figura 41 - Influência do número de partícula na simulação do problema de quebra de barragem utilizando-se o método SPH. O escoamento já atingiu a parede vertical da direita. 


\section{Lista de tabelas}

Tabela 1 - Principais atributos associados às partículas no método MPS.

Tabela 2 - Determinação do raio de vizinhança ( $l_{0}$ é a distância média entre as partículas na configuração inicial).

Tabela 3 - Principais atributos associados às partículas no método SPH.

Tabela 4 -Valores da constante $k$ para cada uma das funções de suavização.

Tabela 5 - Condições iniciais do problema do tubo de choque.

Tabela 6 - Número de partículas de fluido em função do espaçamento 